\title{
AVALIAÇÃO DE SEIS ANOS DE FRATURAS CERVICAIS SUBAXIAIS
}

\author{
EVALUATION OF SIX YEARS OF CERVICAL SUB-AXIAL FRACTURES \\ EVALUACIÓN DE SEIS AÑOS DE FRACTURAS CERVICALES SUBAXIALES
}

Leonardo Franco Pinheiro Gaia1 ${ }^{1}$ Helder de Souza Miyahara1', Olavo Biraghi Letalf², Alexandre Sadao lutaka², Alexandre Fogaça Cristante², Ivan Dias Rocha², Raphael Martus Marcon ${ }^{2}$, Reginaldo Perilo Oliveira ${ }^{3}$, Tarcísio Eloy Pessoa de Barros Filho 4

\begin{abstract}
RESUMO
Objetivo: Avaliar retrospectivamente os fatores relacionados ao trauma cervical, segundo o tipo de fratura baseado na classificação AO. Levando-se em consideração aspectos etiológicos e epidemiológicos do evento. Métodos: Foram analisados retrospectivamente prontuários de pacientes com fratura cervical, no período de 2004 a 2009. Estudou-se as fraturas subaxiais (C3-C7), por se enquadrarem em apenas uma classificação (AO). Usou-se radiografias frente e perfil, e tomografias para dividir as fraturas em A (compressão), B (distração) e C (rotação), de acordo com o padrão apresentado. Analisou-se os seguintes parâmetros: sexo, idade, classificação AO, mecanismo de trauma, presença de déficit neurológico, e tratamento cirúrgico ou conservador. Resultados: Foram analisados 264 prontuários, sendo 216 pacientes do sexo masculino e 48 femininos. A média de idade destes pacientes foi de 38,55 anos. O mecanismo de trauma mais comum das fraturas cervical subaxiais foi o acidente automobilístico com 84 casos. Quanto ao tipo de fratura pela classificação AO, a mais frequente foi do tipo B. Dos casos avaliados, 136 pacientes apresentaram déficit neurológico, parcial ou completo. O tratamento cirúrgico foi realizado em 166 casos. Conclusão: Os dados apresentados demonstram que as fraturas cervicais representam um importante problema para a saúde no Brasil, pública e privada. A prevenção das fraturas cervicais apresenta a melhor relação custo benefício na abordagem destas lesões.
\end{abstract}

Descritores: Fraturas da coluna vertebral; Traumatismo da medula espinal; Vértebras cervicais; Estudos retrospectivos.

\begin{abstract}
Objective: Retrospectively assess the factors related to cervical trauma, according to the type of fracture based on the AO classification, taking into account etiological and epidemiological aspects of the event. Methods: Records of patients with cervical fractures were retrospectively reviewed, from 2004 to 2009. The sub-axial fractures (C3-C7) were studied because they fit into only one classification (AO). Frontal and lateral $x$-rays were used as well as CTs to divide the fractures into A (compression), $B$ (distraction) and $C$ (rotation), in accordance with the presented pattern. The following parameters were assessed: gender, age, AO classification, trauma mechanism, presence of neurological deficit, and conservative or surgical treatment. Results: The study included 264 records from the spine, where 216 patients were male and 48 female. The average age of these patients was 38.55 years. The most common mechanism of injury of the cervical sub-axial fractures was car accident with 84 cases. Regarding the type of fracture by the AO classification, the most frequent was type B. Out of the assessed cases, 136 patients presented complete or partial neurological deficit. The surgical treatment was performed in 166 cases. Conclusion: It is clear, based on the data presented, that cervical fractures are a major problem to the public and private health in Brazil. The prevention of the cervical fractures presents the most cost-effective approach to these injuries.
\end{abstract}

Keywords: Spinal fractures; Spinal cord injuries; Cervival vertebrae; Retrospective studies.

\section{RESUMEN}

Objetivo: Evaluar retrospectivamente los factores relacionados al trauma cervical, según el tipo de fractura, basándose en la clasificación AO, considerandose los aspectos etiológicos y epidemiológicos del evento. Métodos: Fueron analizadas retrospectivamente las historias clínicas de pacientes con fractura cervical, desde el 2004 hasta el 2009. Se estudiaron las fracturas subaxiales (C3-C7), ya que éstas se encuadran en apenas una clasificación (AO). Se utilizaron radiografías en las incidencias frontal y lateral y tomografía computarizada, para dividir las fracturas en A (compresión), B (distracción) y C (rotación), de acuerdo con el patrón de presentación. Se analizaron los siguientes parámetros: sexo, edad, clasificación AO, mecanismo de trauma, presencia de déficit neurológico y tratamiento quirúrgico o conservador. Resultados: Fueron analizadas 264 historias clínicas, siendo 216 pacientes hombres e 48 mujeres. La media de edad de éstos pacientes fue de 38,55 años. El mecanismo de trauma más común de las fracturas cervicales subaxiales fue el accidente automovilístico, con 84 casos. En cuanto al tipo de fractura según la clasificación AO, la más frecuente fue el tipo B. De los casos evaluados, 136 pacientes presentaron déficit neurológico parcial o completo. El tratamiento quirúrgico fue realizado en 166 casos. Conclusión: Queda claro que las fracturas cervicales representan un importante problema para la salud pública y privada en Brasil. La prevención de fracturas cervicales presenta la mejor relación costo beneficio en el abordaje de éstas lesiones.

Descriptores: Fracturas de la columna vertebral; Traumatismos de la médula espinal; Vertebras cervicales; Estudios retrospectivos.

1. Médico Residente do Grupo de Coluna do Instituto de Ortopedia eTraumatologia do Hospital das Clínicas da Faculdade de Medicina da Universidade de São Paulo - São Paulo, SP, Brasil. 2. Médico Assistente do Grupo de Coluna do Instituto de Ortopedia eTraumatologia do Hospital das Clínicas da Faculdade de Medicina da Universidade de São Paulo - São Paulo, SP, Brasil. 3. Médico Chefe do Grupo de Coluna do Instituto de Ortopedia eTraumatologia do Hospital das Clínicas da Faculdade de Medicina da Universidade de São Paulo - São Paulo, SP, Brasil. 4. ProfessorTitular do Departamento de Ortopedia eTraumatologia da Faculdade de Medicina da Universidade de São Paulo - São Paulo, SP, Brasil.

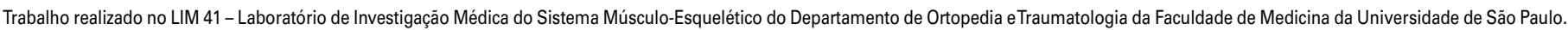
Correspondência: Rua Dr. Ovídio Pires de Campos, 333, 2ªndar, Bairro: Cerqueira César, São Paulo, SP, Brasil. CEP: 05403 - 010. E-mail: leonardo_gaia@uol.com.br 


\section{INTRODUÇÃO}

Os traumatismos cranioencefálicos e do esqueleto são as lesões mais comuns nos pacientes politraumatizados. A prevalência destas lesões é semelhante e em geral acima de 78\%, sendo que as lesões musculoesqueléticas são quatro vezes mais frequentes que as abdominais e duas vezes mais frequentes que as torácicas ${ }^{1}$.

As lesões da coluna vertebral por sua vez ocorrem com menor frequencia que as do esqueleto apendicular estando presente apenas em $6 \%$ dos pacientes politraumatizados. No entanto, quase metade destes pacientes apresentaram algum tipo de lesão neurológica associada e com relação às fraturas cervicais, foco deste estudo, sua prevalência varia em torno de 2 a $6 \%$ dependendo do serviço $0^{2,3}$.

Várias características, tanto do indivíduo como do mecanismo de trauma, podem influenciar no risco de uma fratura cervical. 0 maior risco encontra-se naqueles pacientes que manifestam um déficit neurológico focal (20\%). Outras características relacionadas com risco aumentado são: idade maior que 50 anos, mecanismos de alta energia e a presença de traumatismo cranioencefálico TCE associado ${ }^{3}$. O mesmo mecanismo pode ter diferentes valores no risco de fratura. Por exemplo, em traumas de baixa energia como queda da própria altura, o risco de fraturas em pacientes abaixo de 50 anos é de $0,04 \%$, o mesmo tipo de trauma em pacientes acima de 50 anos eleva este número para $0,5 \%$, ou seja, 10 vezes maior que nas pessoas mais jovens ${ }^{3,4}$.

Os mecanismos de trauma que levam a uma fratura cervical são complexos e não são claramente esclarecidos. Mecanismos similares podem resultar em padrões diferentes de fratura. Em conjunto com a magnitude e direção das forças causadoras da lesão, a orientação da coluna no momento do trauma e estruturas predispostas podem influenciar nos diferentes tipos de fratura ${ }^{5,6}$.

As fraturas cervicais podem ser divididas anatomicamente em coluna cervical alta, formada pelo occipício, atlas(C1) e áxis(C2), e coluna cervical baixa, de C3 a C7. A prevalência das lesões na coluna cervical alta tem uma distribuição bimodal. Elas são encontradas na infância e na população acima dos 60 anos. Na população pediátrica, os acidentes automotivos são a principal causa, já nos idosos, a queda da própria altura é o mecanismo mais comum. Adultos entre estes dois grupos tendem a sofrer lesões da coluna cervical baixa ${ }^{7-9}$.

As lesões do complexo C1-C2 correspondem a 69,8\% das patologias traumáticas cervicais nos idosos, geralmente decorrente de quedas. Algumas explicações para este fenômeno incluem a fragilidade óssea, aumento do estresse na coluna cervical alta devido a espondilose da coluna cervical baixa e o aumento da propensão a queda por deterioração do aparelho locomotor associada à perda do equilíbrio decorrente da idade. A baixa energia do trauma, no entanto, está relacionada a pequenos índices de lesão neurológica nestes pacientes ${ }^{10}$.

Nos adultos jovens, a maioria dos pacientes são do sexo masculino. Os mecanismos mais comuns deste grupo são acidentes automotores, quedas de altura, acidentes com arma de fogo e lesões durante prática esportiva ${ }^{11}$. A maioria dos traumas na coluna cervical desta população ocorre na coluna subaxial, e geralmente estão associados a traumas de grande energia, lesões graves no crânio, déficit neurológico. Traumas na coluna cervical alta nestes indivíduos estão associados a déficit neurológicos severos, com altos índices de morbidade e mortalidade, decorrentes do TCE ou de complicações respiratórias ${ }^{3}$.

O traumatismo raquimedular decorrente destas fraturas tem um alto custo econômico para sociedade. Embora seja difícil realizar esta medida quantitativamente, o maior gasto se deve a perda de vários anos de trabalho produtivo associado a maior sobrevida destes pacientes devido aos avanços na reabilitação oferecidas fazendo com que a estimativa de vida destes pacientes chegue próximo ao normal12. O gasto médico estimado em toda a vida do lesado medular varia de 630.000 a 970.000 dólares por paciente. Apesar da tetraplegia representar apenas 10\% dos pacientes, ela é responsável por $80 \%$ dos gastos médicos, enquanto a paraplegia é responsável por 4\% dos gastos e as lesões incompletas por aproximadamente $15 \%$, segundo estatísticas norte americanas ${ }^{13}$.

A prevenção destes tipos de lesões oferece o melhor retorno dentre as formas de intervenção no intuito de diminuir os gastos médicos e sociais. Estratégias para prevenir ou minimizar as perdas funcionais incluem modificar fatores de risco, alterar mecanismos causadores ou desencadeantes de lesão e interromper as respostas biológicas deletérias a medula ${ }^{14,15}$.

O objetivo do presente trabalho é avaliar retrospectivamente os fatores relacionados ao trauma cervical, segundo o tipo de fratura baseado na classificação AO, levando-se em consideração aspectos etiológicos e epidemiológicos do evento.

\section{MÉTODOS}

Foram analisados retrospectivamente prontuários de pacientes com fratura cervical tratados no Instituto de Ortopedia e Traumatologia do Hospital das Clínicas de São Paulo (IOT-HC-FMUSP), no período de 2004 a 2009.

Estudou-se as fraturas subaxiais (C3-C7), por se enquadrarem em apenas uma classificação (AO). Usou-se radiografias frente e perfil, e tomografias para dividir as fraturas em A (compressão), B (distração) e C (rotação), de acordo com o padrão apresentado.

Analisou-se os seguintes parâmetros: sexo, idade, classificação $A O$, mecanismo de trauma, presença de déficit neurológico e tratamento cirúrgico ou conservador.

Pacientes com mais de um tipo de fratura pela classificação usada, considerou-se apenas a fratura mais grave para facilitar a coleta de dados.

Os mecanismos de trauma foram divididos em queda de altura, acidente automobilístico (carro, moto e bicicleta), atropelamento, queda da própria altura, mergulho em água rasa e outros, nos quais se inclui agressão física, tentativa de auto extermínio, ferimento por arma de fogo, arma branca e trauma direto.

Quanto ao déficit neurológico, observou-se a presença ou ausência deste, sem diferenciá-lo quando encontrado em parcial ou completo. O mesmo ocorreu para o tratamento entre cirúrgico e conservador, sem englobar técnicas e vias utilizadas.

\section{RESULTADOS}

Foram analisados 264 prontuários do ambulatório de coluna do IOT, sendo 216 pacientes do sexo masculino $(81,8 \%)$ e 48 feminino mulheres (18,2\%). A média de idade destes pacientes foi de 38,55 anos, sendo que o paciente mais idoso com 85 anos e a mais jovem com seis anos de idade.

O mecanismo de trauma mais comum das fraturas cervical subaxiais foi o acidente automobilístico totalizando os 264 pacientes do estudo (Tabela 1).

Quanto ao tipo de fratura pela classificação $A O$, a mais frequente foi do tipo B, com 120 casos (45,4\%), seguido pelo tipo A com 86 casos $(32,6 \%)$ e o tipo $\mathrm{C}$ com 58 casos $(22 \%)$.

Nas fraturas tipo A $(n=86)$, o mecanismo de trauma mais comum foi queda de altura, responsável por 25 casos (29\%), seguido por acidente automobilístico, com 23 casos (26,7\%) e mergulho em água rasa, com 11 casos (12,8\%) (Tabela 2).

Tabela 1. Mecanismos de trauma.

\begin{tabular}{c|c|c}
\hline Mecanismo de trauma & $\mathbf{2 6 4}$ & $\%$ \\
\hline Acidente automobilístico & 84 & 31,8 \\
\hline Queda de altura & 83 & 31,4 \\
\hline Queda da própria altura & 27 & 10,2 \\
\hline Atropelamento & 25 & 9,4 \\
\hline Mergulho em água rasa & 18 & 6,8 \\
\hline Outras causas & 27 & 10,2 \\
\hline
\end{tabular}

Tabela 2. Mecanismo de trauma das fraturas Tipo A.

\begin{tabular}{c|c|c}
\hline Mecanismo de trauma Tipo A & $\mathbf{8 6}$ & $\%$ \\
\hline Queda de altura & 25 & 29 \\
\hline Acidente automobilístico & 23 & 26,7 \\
\hline Mergulho em água rasa & 11 & 12,8 \\
\hline Atropelamento & 9 & 10,4 \\
\hline Queda da própria altura & 7 & 8,23 \\
\hline Outras causas & 11 & 12,8 \\
\hline
\end{tabular}


Nas fraturas do tipo B $(n=120)$, houve 40 casos de queda de altura (33,3\%), 36 casos de acidente automobilístico (30\%) e 19 casos de queda da própria altura (15,8\%) (Tabela 3).

Nas fraturas tipo C ( $n=58)$, o acidente automobilístico foi o principal causador, com 25 casos (43,1\%), queda de altura com 18 casos (31\%), seguido por atropelamento com seis casos (10,4\%) (Tabela 4).

Os demais casos de cada fratura, por corresponderem a porcentagens menores, serão apresentados nas tabelas das fraturas $A, B$ e C.

Dos 264 casos avaliados, 136 pacientes apresentaram déficit neurológico (51,51\%), parcial ou completo. O restante 128 pacientes $(48,49 \%)$ não apresentou déficit em nenhum momento da evolução.

Dentre os pacientes com fratura tipo A, 50\% apresentou algum déficit neurológico $(n=86)$, nos do tipo B, $55 \%(n=120)$ e os do tipo C, 46,55\% $(n=58)$ (Tabela 5).

O tratamento cirúrgico foi realizado em 166 casos $(62,9 \%)$; o tratamento conservador em 97 pacientes $(36,7 \%)$ e houve um óbito antes de qualquer tratamento.

Tabela 3. Mecanismo de trauma das fraturas Tipo B.

\begin{tabular}{c|c|c}
\hline Mecanismo de trauma Tipo B & $\mathbf{1 2 0}$ & $\%$ \\
\hline Queda de altura & 40 & 33,3 \\
\hline Acidente automobilístico & 36 & 30,5 \\
\hline Queda da própria altura & 19 & 15,8 \\
\hline Atropelamento & 10 & 8,3 \\
\hline Mergulho em água rasa & 5 & 4,16 \\
\hline Outras causas & 10 & 8,3 \\
\hline
\end{tabular}

Tabela 4. Mecanismo de trauma das fraturas Tipo $\mathrm{C}$.

\begin{tabular}{c|c|c}
\hline Mecanismo de trauma das fraturas Tipo C & $\mathbf{5 8}$ & $\%$ \\
\hline Acidente automobilístico & 25 & 43,1 \\
\hline Queda de altura & 18 & 31 \\
\hline Atropelamento & 6 & 10,4 \\
\hline Mergulho em água rasa & 2 & 3,44 \\
\hline Queda da própria altura & 1 & 1,73 \\
\hline Outras causas & 6 & 10,4 \\
\hline
\end{tabular}

Tabela 5. Classificaçao do déficit neurológico.

\begin{tabular}{c|c|c|c|c}
\hline Déficit neurológico & Sim & $\%$ & Não & $\%$ \\
\hline A & 43 & 50 & 43 & 50 \\
\hline B & 66 & 55 & 54 & 45 \\
\hline C & 27 & 46,55 & 31 & 53,44 \\
\hline
\end{tabular}

\section{DISCUSSÃO}

A partir dos dados obtidos avaliamos que as fraturas cervicais têm um impacto importante na vida da população economicamente ativa, em especial dos pacientes do sexo masculino.

O trauma cervical, com consequente fratura e déficit neurológico, ocorre predominantemente em adultos jovens, em uma pro- porção de $4: 1^{16}$. No presente estudo foram 216 casos do gênero masculino e 48 feminino, o que se aproxima bastante das estatísticas da literatura. A média de idade dos 264 pacientes foi de 38,55 anos, com 154 homens entre os 20 e 50 anos e 27 mulheres nesta mesma faixa etária, que coincide com a faixa etária dos artigos epidemiológicos publicados em outros países.

A causa mais comum de lesão traumática da medula espinhal é acidente automobilístico, mais de 50\% em um levantamento americano ${ }^{17}$. No nosso estudo este tipo de trauma foi o principal responsável por casos de fratura cervical. Queda de altura responde por 31,4\%, e também se encontra em segundo lugar na casuística dos EUA, com 25\%. O que difere são as terceiras e quarta causas enquanto no trabalho dos EUA temos ferimento por arma de fogo com $15 \%$ e lesões em atividades esportivas com $10 \%$, muito provavelmente pela maior militarização daquela população e práticas de esportes com maior contato físico como futebol americano, na nossa avaliação, estas causas são ocupadas por queda da própria altura $(10,2 \%)$, em especial nos pacientes idosos, e atropelamento $(9,4 \%)$.

Com relação ao déficit neurológico, parcial ou completo, dos pacientes com fratura tipo A, 43 o apresentaram, correspondendo a $50 \%$. Nas tipo B, o déficit ocorreu em 66 pacientes, ou $55 \%$ dos casos. Em fraturas do tipo C, 46,55\% tinham déficit neurológico $(n=27)$. A porcentagem entre os três tipos de fratura apresentou pequena variação, embora as fraturas apresentem energia e mecanismo de trauma diferentes. Apesar da tipo $\mathrm{C}$ teoricamente ser a mais grave, este tipo apresentou a menor incidência de déficit neurológico. Estes dados demonstram que, provavelmente, existem outros fatores associados à presença ou ausência de lesão neurológica nas fraturas cervicais como, por exemplo, diâmetro do canal e espondilose cervical ${ }^{18,19}$

O tratamento cirúrgico foi realizado em 166 pacientes, levando-se em consideração os critérios de instabilidade da fratura e o déficit neurológico, que mesmo parcial, também é indicação no nosso serviço.

\section{CONCLUSÃO}

As fraturas cervicais representam um importante problema para a saúde no Brasil. Acometem a população economicamente ativa na sua faixa etária mais produtiva da vida, com consequências, às vezes, irreversíveis para os pacientes e suas famílias.

O mecanismo de trauma mais comum das fraturas cervical subaxiais foi o acidente automobilístico. Quanto ao tipo de fratura pela classificação $\mathrm{AO}$, a mais frequente foi do tipo B. Dos casos avaliados, a maioria (136 pacientes) apresentaram déficit neurológico, parcial ou completo. O tratamento cirúrgico foi realizado em 166 casos.

Conclui-se que a prevenção das fraturas cervicais apresenta a melhor relação custo benefício na abordagem destas lesões. Estratégias para diminuir os números de trauma e conscientização dos indivíduos quanto aos fatores de riscos e mecanismos têm que ser constantemente estudados e divulgados. Ainda assim, o sucesso destas medidas é difícil de ser atingido e quantificado.

\section{REFERÊNCIAS}

1. Riska EB, von Bonsdorff $H$, Hakkinen $S$, Jaroma $H$, Kiviluoto $O$, Paavilainen T. Primary operative fixation of long bone fractures in patients with multiple injuries. J Trauma. 1977:17(2):111-21.

2. Burney RE, Maio RF, Maynard F, Karunas R. Incidence, characteristics, and outcome of spinal cord injury at trauma centers in North America. Arch Surg. 1993;128(5):596-9.

3. Blackmore CC, Emerson SS, Mann FA, Koepsell TD. Cervical spine imaging in patients with trauma: determination of fracture risk to optimize use. Radiology. 1999;211(3):759-65

4. Bub LD, Blackmore CC, Mann FA, Lomoschitz FM. Cervical spine fractures in patients 65 years and older: a clinical prediction rule for blunt trauma. Radiology. 2005:234(1):143-9.

5. Nightingale RW, McElhaney JH, Richardson WJ, Myers BS. Dynamic responses of the head and cervical spine to axial impact loading. J Biomech. 1996;29(3):307-18.

6. Nightingale RW, McElhaney JH, Richardson WJ, Best TM, Myers BS. Experimental impact injury to the cervical spine: relating motion of the head and the mechanism of injury. J Bone Joint Surg Am. 1996;78(3):412-21.

7. McGrory BJ, Klassen RA, Chao EY, Staeheli JW, Weaver AL. Acute fractures and dislocations of the cervical spine in children and adolescents. J Bone Joint Surg Am. 1993:75(7):988-95

8. Sherk HH, Nicholson JT, Chung SM. Fractures of the odontoid process in Young children. J Bone Joint Surg Am. 1978;60(7):921-4.

9. Alker GJ Jr, Oh YS, Leslie EV. High cervical spine and craniocervical junction injuries in fatal traffic accidents: a radiological study. Orthop Clin North Am. 1978;9(4):1003-10.

10. Spivak JM, Weiss MA, Cotler JM, Call M. Cervical spine injuries in patients 65 and older Spine (Phila Pa 1976). 1994;19(20):2302-6.

11. Katzberg RW, Benedetti PF, Drake CM, Ivanovic M, Levine RA, Beatty CS, et al. Acute cervical spine injuries: prospective MR imaging assessment at a level 1 trauma center. Radiology. 1999;213(1):203-12.

12. Wright M, Rivara FP, Ferse D. Evaluation of the Think First head and spinal cord injury prevention program. Inj Prev. 1995;1(2):81-5.

13. DeVivo MJ. Causes and costs of spinal cord injury in the United States. Spinal Cord. 1997:35(12):809-13

14. Rivara FP, Grossman DC, Cummings P. Injury prevention. First of two parts. N Engl J Med. 1997;337(8):543-8.

15. Rivara FP, Grossman DC, Cummings P. Injury prevention. Second of two parts. N Engl J Med. 1997:337(9):613-8

16. Parsons KC. The impact of spinal cord injury on long-term survival. J Insur Med. 1991;23(4):227.

17. Woodruff BA, Baron RC. A description of nonfatal spinal cord injury using a hospital-based registry. Am J Prev Med. 1994;10(1):10-4.

18. Bosch PP, Vogt MT, WardWT. Pediatric spinal cord injury without radiographic abnormality (SCIWORA): the absence of occult instability and lack of indication for bracing. Spine (Phila Pa 1976). 2002;27(24):2788-800

19. Defino HLA. Lesões Traumáticas da coluna vertebral. São Paulo: Bevilacqua Editora; 2006 